\title{
Correction to: Non-Melanoma Skin Cancer and Cutaneous Melanoma
}

Othon Papadopoulos, Nikolaos A. Papadopulos, and Grigorios Champsas

\section{Correction to: O. Papadopoulos, N. A. Papadopulos, G. Champsas (eds.), Non-Melanoma Skin Cancer and Cutaneous Melanoma, https://doi.org/10.1007/978-3-030-18797-2}

The affiliation of the editor in the Front matter was published with an error. This has been corrected now as mentioned below.

Othon Papadopoulos, MD, PhD, Department of Plastic, Reconstructive and Aesthetic Surgery, National and Kapodistrian University of Athens, Medical School, Athens, Greece.

The role of the editor Dr. Nikolaos Papadopoulos was inadvertently mentioned in the contributors list. This was also removed for consistency purpose.

The updated online version of this book can be found at https://doi.org/10.1007/978-3-030-18797-2 